\title{
Investigación
}

\section{Compromiso cardiovascular en pacientes con Síndrome Inflamatorio Pediátrico Multisistémico, asociado a infección por SARS-CoV- 2}

\author{
Patricia Álvarez ${ }^{1,2}$, Valeria Acevedo ${ }^{1,2}$, María Lidia Valenzuela $^{1}$, Vicente Montes ${ }^{a}$, Patricia Aroca ${ }^{1}$, Carla García ${ }^{1,2}$, Carolina Campos ${ }^{1,2}$, Katherine $^{2}$ \\ Koch $^{1}$, Soledad González ${ }^{1}$, Cecilia Poli ${ }^{3}$, Patricia Verdugo ${ }^{2,4}$, Fernanda Cofré ${ }^{5}$, Jorge Mackenney ${ }^{2}$, Lorena Tapia $^{2}$. \\ 1. Servicio de Cardiología y Cardiocirugía. Hospital Roberto del Río. Santiago, Chile \\ 2. Departamento de Pediatría, Campus Norte, Facultad de Medicina, Universidad de Chile, Santiago, Chile. \\ 3. Unidad de Inmunología-Reumatología. Hospital Roberto del Río. Santiago, Chile. \\ 4. Unidad de Hematología. Hospital Roberto del Río. Santiago, Chile. \\ 5. Unidad de Infectología. Hospital Roberto del Río. Santiago, Chile. \\ a. Alumno de Medicina, Facultad de Medicina. Clínica Alemana- Universidad del Desarrollo.
}

Introducción: El Síndrome Inflamatorio Pediátrico Multisistémico (PIMS) ha emergido como una nueva enfermedad en niños, secundaria a infección por SARS- CoV-2. Se caracteriza por presentar compromiso multiorgánico con parámetros inflamatorios elevados y manifestaciones clínicas graves, siendo el corazón el órgano más severamente comprometido.

Objetivo: Describir las características clínicas y de laboratorio de 23 pacientes con diagnóstico de PIMS con compromiso cardiovascular hospitalizados en un centro único.

Método: Se efectuó un estudio retrospectivo analizando los hallazgos clínicos y de laboratorio junto a las manifestaciones cardiovasculares que presentaron estos pacientes.

Resultados: 23/29 pacientes con PIMS (78\%) presentaron manifestaciones digestivas y mucocutáneas. Las manifestaciones cardiovasculares fueron: Síndro- me Kawasaki y "Kawasaki like" sin compromiso coronario en $15 / 23(65 \%)$ y con compromiso coronario en $3(13 \%)$. Shock en 9 pacientes (39\%), injuria miocárdica- miocarditis en 8 (35\%) y derrame pericárdico en 13 (56\%). Trastornos del ritmo cardíaco se observaron en 6 pacientes (26\%). La terapia más utilizada fue inmunoglobulina y corticoides. $18 / 23$ requirieron manejo en unidades de intermedio y/o intensivo. Un $70 \%$ de los pacientes se recuperó del compromiso cardiovascular antes del alta.

Conclusión: El compromiso cardiovascular en PIMS es la complicación más frecuente de esta enfermedad, que se acompaña de manifestaciones inmunológicas y hematológicas graves lo que hace necesario un tratamiento multidisciplinario para un mejor manejo de estos pacientes.

Palabras clave: COVID-19; aneurisma de arteria coronaria; Kawasaki; injuria miocárdica.

Correspondencia:

Dra. Patricia Álvarez Zenteno

pazenteno@yahoo.com 


\section{Cardiovascular involvement in pediatric patients with SARS-CoV-2 associated to multisystemic inflammatory syndrome.}

Introduction: Pediatric Multisystemic Inflammatory Syndrome (PIMS) has emerged as a new disease in children, secondary to SARS- CoV-2 infection. It is characterized by multi-organ involvement with elevated inflammatory parameters and severe clinical manifestations, the heart being the organ most severely involved.

Objective: to describe the clinical and laboratory characteristics of 23 patients diagnosed with PIMS with cardiovascular involvement hospitalized in a single center.

Method: We conducted a retrospective study in which we analyzed the clinical and laboratory findings along with the cardiovascular manifestations presented by these patients.

Results: 23/29 patients with PIMS and cardiovascular involvement were selected, $78 \%$ had digestive and mucocutaneous manifestations. Cardiovascular manifestations consisted of Kawasaki- Kawasaki like syndrome without coronary involvement in $15 / 23(65 \%)$ and coronary involvement in $3(13 \%)$. Nine patients developed shock (39\%), 8 (35\%) myocardial injury in and $13(56 \%)$ pericardial effusion.. Heart rhythm disorders were observed in 6 patients (26\%). The main therapy was immunoglobulin and corticosteroids. $18 / 23$ required management in intermediate and/or intensive care unit. $70 \%$ of patients recovered from cardiovascular involvement before discharge.

Conclusion: Cardiovascular involvement in PIMS is the most frequent complication of this disease, but it is associated with severe immunological and hematological manifestations, which makes necessary a multidisciplinary treatment for a better management

Key words: COVID-19; coronary artery aneurysm,; Kawasaki disease; myocardial injury. 


\section{Introducción:}

Desde su descripción inicial en diciembre de 2019 en Wuhan, China, la enfermedad conocida como COVID-19 producida por el virus SARS-CoV-2, se ha diseminado a nivel mundial, siendo declarada pandemia por la OMS en marzo del $2020^{1}$ y afectando, hasta la fecha, a más de 17 millones de personas en el mundo.

Inicialmente, se consideró como una infección leve $e^{2,3}$ para los niños, en comparación a los adultos. Sin embargo, con la aparición de un nuevo cuadro, denominado Síndrome Inflamatorio Pediátrico Multisistémico Asociado a COVID-19 (PIMS), esto se ha modificado. Los criterios diagnósticos para dicho síndrome se encuentran aún en discusión, siendo los más utilizados los del Royal College of Pediatrics and Child Health ${ }^{4}$, Centers for Disease Control (CDC $)^{5}$ y de la Organización Mundial de la Salud $(\mathrm{OMS})^{6}$.

Se caracteriza por un cuadro febril con importante inflamación sistémica y que afecta uno o más órganos en pacientes expuestos a este virus. El compromiso cardiovascular comanda el pronóstico y la morbimortalidad en niños y está caracterizado por la presencia de disfunción miocárdica, dilatación y aneurismas coronarios, alteraciones de la conducción eléctrica y, con menor frecuencia, presencia de derrame pericárdico y compromiso valvular $^{7-9}$.

La etiología aún no ha sido aclarada y se ha planteado como un fenómeno post infeccioso donde participa una tormenta de citoquinas en un huésped con predisposición individual y genética. En nuestro país los primeros casos de PIMS, comenzaron a aparecer en mayo del 2020 manifestándose como una enfermedad heterogénea con distintos grados de severidad y de secuelas ${ }^{10}$.

El objetivo de este trabajo es describir una serie clínica de 23 pacientes con PIMS con compromiso cardiovascular hospitalizados en un centro único, desde mayo a julio del 2020.

\section{Métodos:}

Se efectuó un estudio retrospectivo de 23 de 29 pacientes con diagnóstico de PIMS y compromiso cardiovascular, ingresados en el Hospital Roberto del Río de Santiago de Chile, entre el 11 de mayo y el 23 de julio del 2020.

Se utilizó la definición del $\mathrm{CDC}^{5}$ para PIMS y se consideró exposición al virus: RT- PCR COVID-19 (+), IgM (+) o IgG (+) para SARS-COV-2 y/o el antecedente de contacto con paciente COVID-19 (+).

Se revisaron las fichas clínicas de los 23 pacientes analizándose edad, género, comorbilidades, presencia de signos y síntomas mucocutáneos, gastrointestinales, cardiológicos, respiratorios y neurológicos. Los exámenes de laboratorio evidenciaban inflamación y compromiso multisistémico como Proteína-c-reactiva (PCR), fibrinógeno, ferritina, dímero D, albúmina, recuentos de glóbulos blancos y de plaquetas.

Para fines de este estudio se clasificaron los pacientes según su forma de presentación en aquellos con síntomas similares a la enfermedad de Kawasaki (típica u atípica) ${ }^{11}$, pacientes con injuria miocárdica-miocarditis, pericarditis y valvulitis y el tercer grupo incluyó los pacientes con shock.

Shock se definió como insuficiencia circulatoria con la presencia de hipotensión arterial y requerimiento de drogas vasoactivas.

Miocarditis fue definido como disfunción miocárdica en el ecocardiograma Doppler color, asociado a elevación de troponinas, pro-BNP y/o a la presencia de arritmia en el electrocardiograma ${ }^{12}$. Compromiso pericárdico se definió por la presencia de derrame pericárdico en la ecocardiografía.

Para la evaluación del compromiso cardiovascular se revisaron los electrocardiogramas de 12 derivaciones, ecocardiogramas Doppler color con técnica convencional y avanzada, y exámenes de laboratorio (troponinas y pro-BNP).

Se usaron las guías de la Asociación Americana de Ecocardiografía para la evaluación de la función miocárdica global y segmentaria ${ }^{13}$. El grado de compromiso de la función sistólica del ventrículo izquierdo (VI) se clasificó en leve, moderada y severa según la fracción de eyección (FE).

La evaluación de las arterias coronarias se realizó con los parámetros de Z-score publicados por Dallaire ${ }^{14}$ y se definió dilatación y aneurisma coronario según la guía de la enfermedad de Kawasaki de la Sociedad Americana de Cardiología 201711. Se analizó además la presencia de derrame pericárdico y compromiso valvular.

Se revisó el tipo de terapia utilizada como también el seguimiento de estos pacientes.

Análisis estadístico: Las variables fueron descritas utilizando frecuencias absolutas y porcentajes para las variables dicotómicas y medianas y rango para análisis no paramétrico. Las comparaciones se realizaron mediante las pruebas $\chi 2$ o U-Mann-Whitney según correspondiere. Se consideró significativo un valor "p" menor a 0,05 .

\section{Resultados:}

De un total de 29 pacientes ingresados en el servicio de 


\section{Figura 1.}

\section{PIMS CV - DISTRIBUCION POR EDAD}

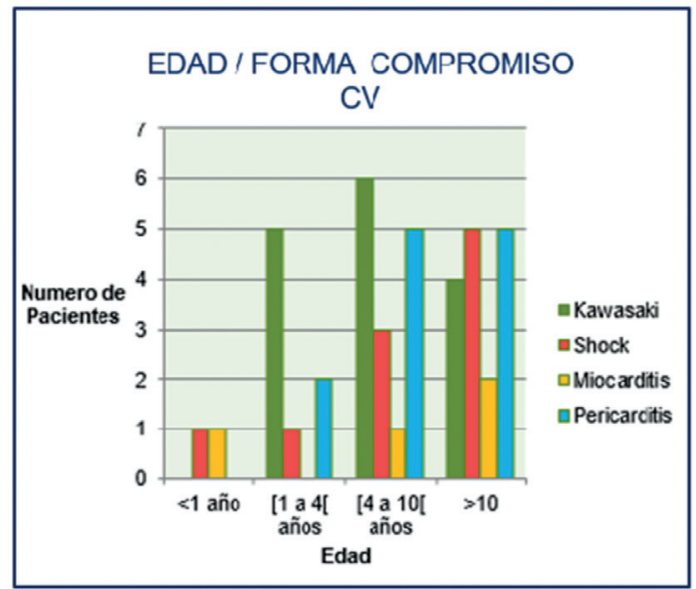

Distribución del tipo de manifestación cardiovascular según edad.

pediatría del Hospital Roberto del Río con diagnóstico de PIMS, 23 presentaron compromiso cardiovascular.

La mediana de edad de este grupo fue de 6,2 años (rango 2 meses a 14 años), 14 de ellos hombres (60\%). 3/23 pacientes presentaban morbilidad previa, 2 con obesidad leve y uno de ellos además asmático. Un lactante de 2 meses, el menor de nuestra serie, con antecedente de ser pre término de 33 semanas y pequeño para la edad gestacional. La distribución del tipo de manifestación cardiovascular según edad se describe en la Figura 1.

Las manifestaciones clínicas según sistema se distribuyeron en digestivas, 18 pacientes (78\%) correspondiendo a diarrea, dolor abdominal y vómitos los síntomas más frecuentes; hubo compromiso mucocutáneo en 18 pacientes (78\%), con exantema e inyección conjuntival como las presentaciones clínicas más frecuentes. El compromiso respiratorio se presentó en 8 pacientes (35\%), de los cuales tres cursaron con neumonía. La cefalea constituyó el síntoma neurológico más frecuente (22\%). Siete pacientes se encontraban aún hospitalizados al momento del cierre del estudio. La mediana de días de hospitalización fue 9 días (rango 5 a 22) y la estadía en la Unidad de Cuidado Intensivo fue 5 días (rango 2-12).

La determinación de PCR para virus SARS-CoV-2 fue positiva en 11 (47\%) pacientes; la serología en $13(70 \%)$ y antecedente de contacto con paciente COVID $19(+)$ en 12 niños. La PCR estaba elevada en el $100 \%$ de los pacientes con rango entre 60 a $408 \mathrm{mg} / \mathrm{L}$, mediana de 228 $\mathrm{mg} / \mathrm{L}$ para un valor normal menor a $5 \mathrm{mg} / \mathrm{L}$. La ferritina estaba elevada en 20/23 (87 \%) y la mediana para el grupo general fue 429 (rango $42-3.805 \mathrm{mg} / \mathrm{ml}$ ).

El dímero D estaba elevado en todos los pacientes: mediana 2.168 (rango 503- $5.975 \mathrm{ng} / \mathrm{ml}$ ). El fibrinógeno estuvo elevado en todos, con mediana de 460,5 (rango 328$1.124 \mathrm{mg} \%$ ). La albúmina plasmática estaba disminuida en 19/21 (90 \%) con una mediana de 2,2 g/dl (rango de $1,7-4,5 \mathrm{~g} / \mathrm{dl})$.

Se observó leucocitosis en 13/23 (57\%) y linfopenia en $9(39 \%)$. Doce pacientes tuvieron recuento de plaquetas menores de 150.000 (52\%) y dos mayores a 600.000.

Del análisis de los marcadores cardíacos, la troponina fue medida en todos los pacientes con rango entre 0,01 a 2,8 $\mathrm{ng} / \mathrm{ml}$. En 10 pacientes (44\%) se encontraba elevada, con mediana de 1,19 (VN < de 0,034 ng/ml). El Pro-BNP se midió en 7, y estaba elevado en cuatro con mediana de $212 \mathrm{pg} / \mathrm{ml}(\mathrm{VN}<125 \mathrm{pg} / \mathrm{ml})$.

Las formas clínicas de presentación cardiovascular fueron: Kawasaki- Kawasaki like sin compromiso coronario en $15 / 23(65 \%)$ y con compromiso coronario en 3 $(13 \%)$. Se presentó shock en 9 pacientes (39\%), injuria miocárdica/miocarditis en 8 (35\%) y pericarditis en 13 (56\%), (Figura 2).

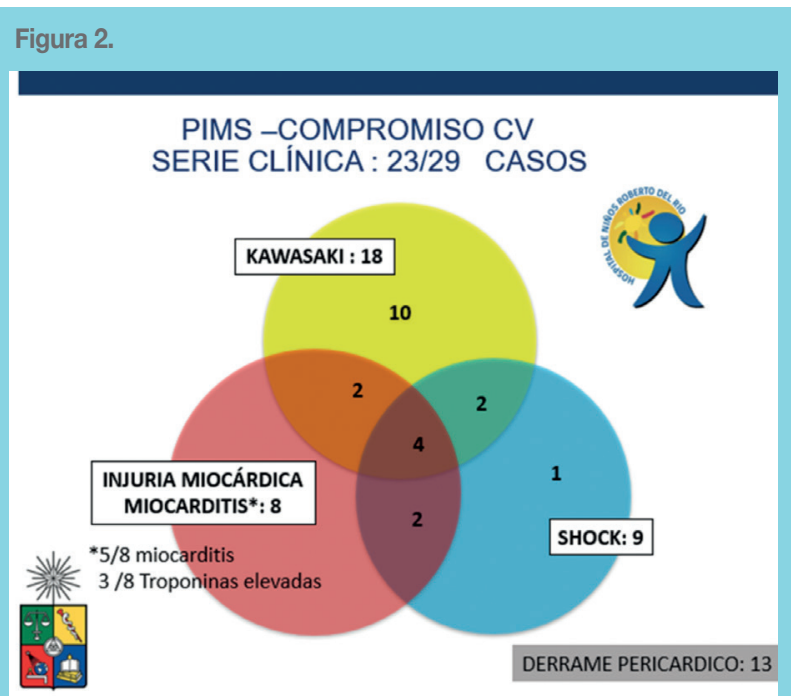

Distribución según forma de compromiso cardiovascular.

Trastornos del ritmo cardíaco se observaron en 6 pacientes (26 \%): 3 con bradicardia sinusal, dos con bloqueo aurículoventricular de primer grado transitorio y un lactante que cursó con miocarditis grave y taquicardia ventricular. 
En cuanto a los hallazgos ecocardiográficos, 8/23 (35\%) presentaron disfunción miocárdica: depresión leve de la FE del VI se observó en 2 pacientes y moderada en 4; disfunción diastólica del VI en 2 y disfunción sistodiastólica del VD en uno. Tres pacientes presentaron compromiso coronario, todos ellos con elementos clínicos de enfermedad de Kawasaki. Un caso presentó dilatación del tronco coronario izquierdo (TCI) (Z-score $+2,23)$ y de la arteria coronaria descendente anterior (DA) (Z-score +2,16). Otro con aneurisma pequeño de la DA (Z-score $+2,8)$. El tercer paciente con compromiso de las tres arterias coronarias, con dilatación del TCI (Z-score $+2,28$ ), aneurisma pequeño de la DA (Z-score $+4,8)$ y de la arteria circunfleja (Z-score+2,6) además de un aneurisma mediano de la arteria coronaria derecha (Z-score $+6,9$ ) (Figura 3). Hubo derrame pericárdico en 13 pacientes, cuya magnitud fluctuó entre laminar y hasta 12 mm., sin compromiso hemodinámico. Se observó Insuficiencia mitral leve en 2 pacientes.

Nueve pacientes presentaron shock, de los cuales seis con elementos clínicos de Kawasaki. Del análisis de este grupo versus el que no tuvo compromiso hemodinámico, la albúmina y la troponina mostraron una diferencia estadísticamente significativa (Tabla 1).

La terapia utilizada fue: inmunoglobulina endovenosa en 20/ 23 (87\%), de los cuales tres recibieron dos dosis. Se administró metilprednisolona a17 /23 (74 \%), drogas

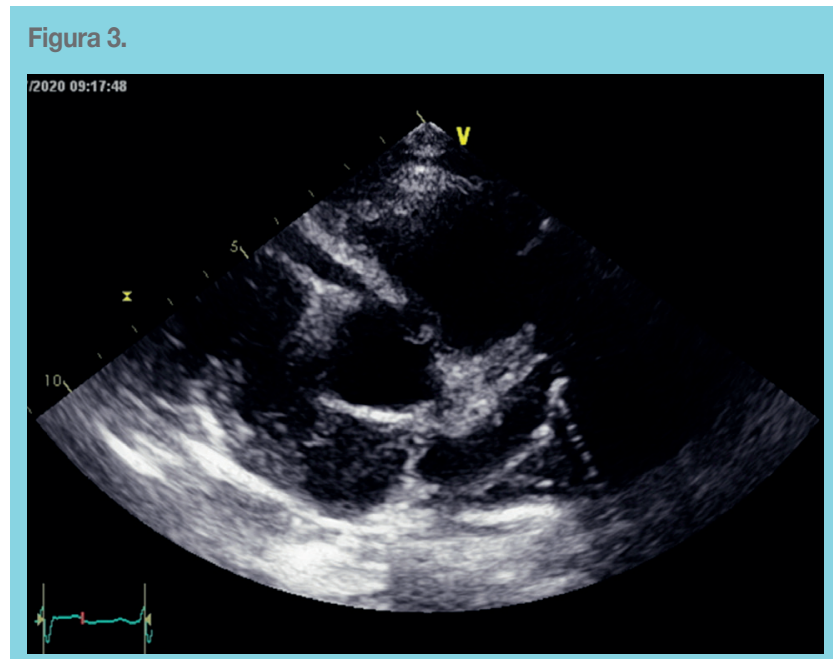

Ecocardiografía, eje corto paraesternal; aneurisma de la arteria coronaria derecha.

vasoactivas a 9/23 (39\%) y se implementó ventilación mecánica en $8 / 23$ (34\%). Recibieron aspirina 22 pacientes $(96 \%)$ y enoxaparina $17 / 23(74 \%)$. En dos pacientes se utilizó tocilizumab y en uno infliximab.

El rango de seguimiento fue entre una a diez semanas. En la evaluación ecocardiográfica se observó mejoría total de la función miocárdica en 6/8 y parcial en dos. De los 13 pacientes con derrame pericárdico, en 8 hubo resolución completa y cinco persisten con derrame pericárdico

\begin{tabular}{|c|c|c|c|}
\hline Hallazgo & SHOCK $(n=9)$ & NO SHOCK $(n=14)$ & $\mathrm{p}$ \\
\hline Edad meses* & $107(2-166)$ & $73,5(23-173)$ & 0,284 \\
\hline Sexo\# & $7(78)$ & $7(50)$ & 0,183 \\
\hline Síntomas gastrointestinales\# & $8(89)$ & $10(71)$ & \\
\hline $\mathrm{PCR}^{*}$ & $204(100,7$ - 400) & $230(60,5-408)$ & 0,728 \\
\hline Ferritina* & $466(42-3805)$ & $361(111-2972)$ & 0,488 \\
\hline Dímero D* & $2191(503-5000)$ & $2137,5(672-5975)$ & 0,528 \\
\hline Fibrinógeno* & $464(328-770)$ & $457(354-1124)$ & 0,688 \\
\hline Albúmina* & $2,1(1,7-2,2)$ & $2,75(1,9-4,5)$ & 0,016 \\
\hline Plaquetas (miles/dL)* & $104(37-233)$ & $146,5(28-649)$ & 0,068 \\
\hline Plaquetopenia $(<150.000) \#$ & $7(78)$ & $7(50)$ & 0,183 \\
\hline Recuento absoluto linfocitos* & $738(99-3200)$ & $1038(242-2125)$ & 0,413 \\
\hline Troponina* & $0,7(0,01-2,8)$ & $0,01(0,01-2,4)$ & 0,020 \\
\hline
\end{tabular}




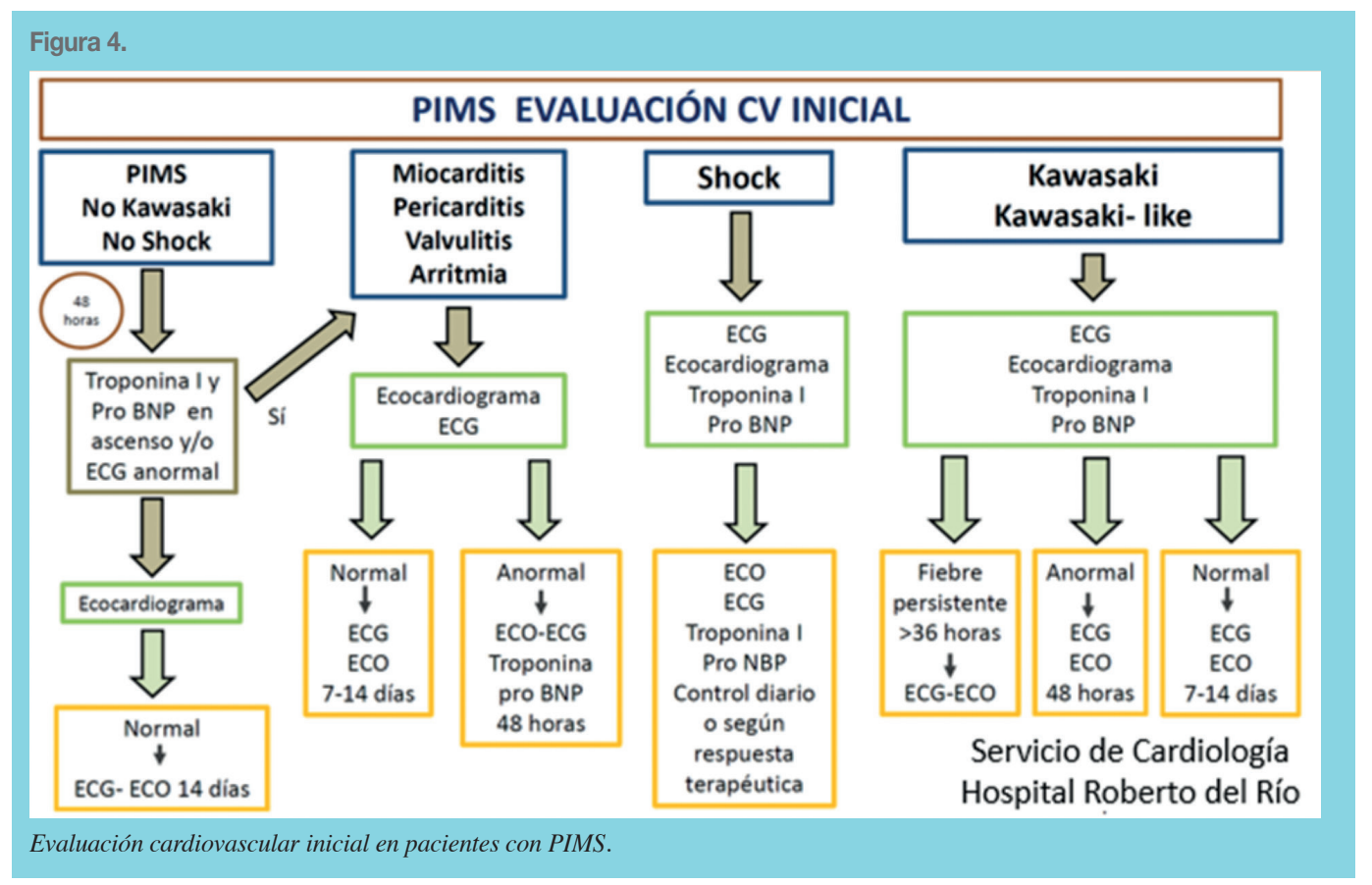

laminar. De los tres pacientes con compromiso coronario, dos normalizaron sus arterias coronarias previo al alta $\mathrm{y}$ el paciente con compromiso global aún persiste hospitalizado en la fase aguda de la enfermedad.

\section{Discusión:}

Desde su aparición en diciembre 2019 la infección por SARS-CoV-2 en humanos ha sido un constante desafío para la comunidad médico-científica. Si bien desde un inicio no se contempló a la población pediátrica como un grupo de riesgo, en los últimos 4 meses se han descrito a nivel mundial un número creciente de casos de PIMS asociado a SARS-CoV-2, en pacientes sin mayores comorbilidades 15,16 .

Al igual como se describe en otras publicaciones ${ }^{16-17}$, en nuestra serie la población afectada fue principalmente escolares y adolescentes (60\% mayor a seis años).

Los menores de 10 años presentaron con mayor frecuencia fenotipo de enfermedad de Kawasaki y en los mayores de 10 años fue más frecuente el shock. El hallazgo cardiovascular más frecuente de esta serie fue la presencia de derrame pericárdico y se vio principalmente en escolares y adolescentes.

El compromiso cardiovascular se presenta de forma heterogénea y con frecuencia las manifestaciones clínicas se superponen lo que dificulta su clasificación. Como una forma de ordenarnos para el enfrentamiento diagnóstico y terapéutico, clasificamos a nuestros pacientes en tres grupos, según la presentación clínica dominante lo que nos permitió definir el esquema terapéutico y de seguimiento (Figura 4).

De las formas clínicas de compromiso cardiovascular, gran parte de nuestros pacientes desarrollaron formas mixtas, de los cuales cuatro pacientes $(18 \%)$ presentaron los tres fenotipos.

El 13,6\% de los pacientes tuvieron dilatación y aneurismas coronarios. Si bien este grupo tenían parámetros inflamatorios elevados, dicha alteración no fue estadísticamente significativa, probablemente, debido al bajo número de pacientes incluidos.

Cabe destacar que el $39 \%$ de nuestros pacientes cursó con deterioro cardiovascular rápidamente progresivo y shock, requiriendo de tratamiento en Unidad de Cuidados Intensivos, reflejando una entidad clínica grave. Por ello, es necesario un alto nivel de sospecha y trasladar a los pacientes a un centro terciario para realizar diagnóstico y tratamiento apropiado.

Los parámetros de laboratorios analizados como PCR, ferritina, dímero D se encontraban severamente elevados en esta serie lo que revela lo grave del compromiso inflamatorio multisistémico de esta enfermedad. El examen más alterado de este grupo fue la PCR, sin embargo, no se relacionó con el agravamiento de la enfermedad como 
tampoco con el desarrollo de compromiso coronario, por lo que la etiología inflamatoria no sería el único factor que determina la severidad de la enfermedad.

La hipoalbuminemia y la troponina fueron los únicos parámetros que diferenciaron aquellos pacientes que evolucionaron con compromiso hemodinámico y shock, lo que podría tener algún valor predictivo del deterioro clínico de los enfermos.

La terapia con inmunoglobulina y corticoides fue la más utilizada, observándose una buena respuesta, Sin embargo, se necesitan más estudios clínicos controlados para establecer el tratamiento óptimo que evite la progresión de la enfermedad y el desarrollo de aneurismas coronarios. A pesar de lo grave del compromiso miocárdico y coro- nario, se observó una buena respuesta a la terapia y en la mayoría de los casos tuvimos resolución precoz del compromiso cardiológico, aunque falta tiempo de seguimiento para determinar si persistirán secuelas. Estamos aún en la etapa aguda de esta nueva enfermedad, quedando todavía varias preguntas por resolver.

En conclusión, el Síndrome Inflamatorio Pediátrico Multisistémico Asociado a COVID-19 constituye una enfermedad nueva, grave, con compromiso multisistémico, por lo que requiere un manejo multidisciplinario conformado por pediatras, intensivistas, infectólogos, inmunólogos, hematólogos y cardiólogos, que en conjunto determinen el mejor esquema diagnóstico, terapéutico y de seguimiento.

\section{Referencias}

1- Organización Mundial de la Salud (OMS), ed. (11 de marzo de 2020). "Alocución de apertura del Director General de la OMS en la rueda de prensa sobre la COVID-19 celebrada el 11 de marzo de $2020 "$ "

2- Ramcharan T, Nolan O, Yi Lai1 C, Prabhu N, Krishnamurthy $\mathrm{R}$ et al. Paediatric Infammatory Multisystem Syndrome: Temporally Associated with SARS CoV 2 (PIMS TS): Cardiac Features, Management and Short Term Outcomes at a UK Tertiary Paediatric Hospital. Pediatr Cardiol. 2020; Jun 12: $1-11$.

3- Pouletty M, Borocco C, Ouldali N et al. Paediatric multisystem inflammatory syndrome temporally associated with SARS-CoV-2 mimicking Kawasaki disease (Kawa-COVID-19): a multicentre cohort. Annals of the Rheumatic Diseases. 2020; 79:999-1006.

4- www.RCPCH.AC.UK/cites/default/file/20-05/COVID-19-Paediatric-multisystem-\% 20 inflammatory \% 20 syndrome -20200501. Pdf.

5- Center of Control Disease. Multisystem Inflammatory Syndrome in Children (MIS-C) Associated with Coronavirus Di- sease 2019 (COVID-19). Official Health Advisory. [Internet]. [citado 25 de julio de 2020]. Disponible en: https://emergency.cdc.gov/han/2020/han00432.asp.

6- World Health Organization. Multisystem inflammatory syndrome in children andadolescents with COVID-19. Published May 15, 2020. Accessed May 22, 2020.https://www.who. int/publications-detail/multisystem-inflammatorysyndromein-children-and-adolescents-withcovid-19.

7- Zachariah P, Johnson C, Halabi K, Ahn D, Sen A et al Epidemiology, Clinical Features, and Disease Severity in Patients With Coronavirus Disease 2019 (COVID-19) in a Children's Hospital in NewYork City, NewYork. JAMA Pediatr. doi:10.1001/jamapediatrics.2020.2430. Published online June 3,2020 .

8- Whittaker E, Bamford A, Kenny J, Kaforou M, Jones C, Shah Pet al. Clinical Characteristics of 58 Children With a Pediatric Inflammatory Multisystem Syndrome Temporally Associated With SARS-CoV-2. JAMA. doi:10.1001/jama.2020.10369 Published online June 8, 2020.

9- Toubiana J, Poirault C, Corsia A, Bajolle F, Fourgeaud J et al. 
Outbreak of Kawasaki disease in children during COVID-19 pandemic: a prospective observational study in Paris, France. medRxiv. Preprint posted May 2020. doi:10.1101/2020.05.10 .20097394 .

10- Cofré F, Izquierdo G, Tapia L, Poli C, Delpiano L, Conca N et al. Recomendaciones de Diagnóstico y Manejo de Síndrome Inflamatorio Multisistémico en contexto pandemia SARSCoV-2. Santiago: Sociedad Chilena de Infectología; 2020.

11- McCrindle B, Rowley A, Newburger J, Burns J, Bolger A, Gewitz $\mathrm{M}$ et al. Diagnosis, treatment, and long-term management of Kawasaki disease: a scientific statement for health professionals from the American Heart Association. Circulation.2017;135(17): e927-e999.

12- Dufort E, Koumans E, Chow E, Rosenthal E, Muse A, Rowlands $\mathrm{J}$ et al. Multisystem Inflammatory Syndrome in Children in New York State. N Engl J Med. 2020; 383:347358. DOI: 10.1056/NEJMoa2021756.

13- Lang R, Badano L, Mor-Avi V, Afilalo J, Armstrong A, Ernande L et al. Recommendations for Cardiac Chamber Quantification by Echocardiography in Adults: an Update from the
American Society of Echocardiography and the European Association of Cardiovascular Imaging. J Am Soc. Echocardiogr. 2015; 28:1-39.

14- Dallaire F, Dahdah N. New equations and critical appraisal of Coronary Artery Z scores in Healthy Children. J American Society of Echocardiography. J Am Soc Echocardiogr. 2011; 24(1): 60-74.

15- Riphagen S, Gomez X, Gonzalez-Martinez C, Wilkinson N, Theocharis P. Hyperinflammatory shock in children during COVID-19 pandemic. Lancet. 2020;395(10237):1607-1608. doi:10.1016/S0140- 6736(20)31094-1.

16- Feldstein L, Rose E, Horwitz S, Collins J. Newhams M, Son $\mathrm{M}$ et al. Multisystem Inflammatory Syndrome in U.S. Children and Adolescents. N Engl J Med. June 2020; DOI: 10.1056/NEJMoa2021680.

17- Belhadjer Z, Méot M, Bajolle F, et al. Acute heart failure in multisystem inflammatory syndrome in children (MIS-C) in the context of global SARS-CoV-2 pandemic. Circulation. 2020; 382:1370-22. 